\title{
Smart Lab with Automated Access
}

\author{
Milan Vishnoi $^{\# 1}$, Kshitiz Saxena ${ }^{\# 2}$, Mohammad Anas ${ }^{\# 3}$, Sachin Singh $^{\# 4}$, Navita Agarwal ${ }^{* 5}$ \\ ${ }^{\# 1,2,3,4}$ Student, Department of Computer Science \& Engineering, MIT, Moradabad, U.P, India \\ ${ }^{*}$ Assistant Professor, Department of Computer Science \& Engineering, MIT, Moradabad, U.P, India
}

\begin{abstract}
This paper deals with the idea of smart labs with automated access utilizing IOT for door unlocking systems and automating the process of switching ON the appliances to provide smartness and automation to our computer labs. It uses a picture capturing technique in an embedded system based on Raspberry Pi. RPi (Raspberry pi) controls the camera for capturing the image and for door unlocking. The camera captures the facial picture and RPi processes the image to the service which recognises the face in the image by comparing it with the images which are stored in the database. If the picture is found in the database then the door lock opens and a node is assigned to the person, the node and nearby fan and light are provided power. The person will be informed by an audio announcement about the assigned node.
\end{abstract}

Keywords - Internet of Things(IOT),Image matching, Cloud Computing, Sensor System, Facial Recognition, Digital camera, Raspberry Pi 3, Person Identification, Smart lab.

\section{INTRODUCTION}

In these modern times, automation is the need of the hour for the development of society as a whole which in turn will help make our labs automated, so the concept of facial recognition to gain access to the node in a lab is an idea which is used to make our labs smart and automated. A facial recognition system is a system which captures facial images and verifies the identity of a person using a digital camera[1]-[3].

The human face assumes an essential part in our social association, passing on individuals' character. Utilizing the human face as a key to biometric confront acknowledgment innovation has gotten tremendous consideration in the previous quite a while because of its potential for a wide assortment of utilizations[5].

A facial acknowledgment framework is a framework which gets facial pictures and confirms the character of a man using a propelled camera. It is an application fit for distinguishing or checking a man from a computerized picture. One approach to do this is by looking at chosen facial components from the picture and a face database[6].

As stood out from other diverse biometrics frameworks utilizing unique mark/palm print and iris, confront face acknowledgement has unmistakable favourable circumstances due to its non-contact handle. Face pictures can be captured from a separation without touching the individual being recognized. Face Recognition is normally utilized as a part of security frameworks and can be contrasted with different biometrics. It has additionally turned out to be mainstream as a commercial recognizable proof. We have also used cloud computing to store the database of images and for facial recognition. In our project we have used the services provided by Amazon Web Services(AWS).

The use of cloud computing enables the system to be scalable[7]. If there are many students and the database of those students cannot be stored on the available SD card, then using of a new SD card would create problem and would be difficult to expand the size but when we use cloud to store our database then the storage capacity can be increased easily because unlimited storage is available in the cloud. One more reason for using cloud computing is that it also prevents us from creating databases on different Raspberry Pi, that is , if there are more than one lab then all the Raspberry Pi systems can access the database stored in the cloud, otherwise there would be a need to create separate databases for each RPi system. 


\section{HARDWARE DESCRIPTION}

\section{A. Raspberry Pi 3 Model B}

In this work we utilized Raspberry Pi 3 Model B. It is a single board PC created in the United Kingdom by the Raspberry Pi establishment. Raspberry Pi has many generations. What we are using here is Pi 3.It replaced Pi 2 model B in February 2016. Raspberry Pi 3 is believed to be approximately $80 \%$ faster than RPi2 in parallelized tasks. Price is in between INR 2800 to INR 4000. It has architecture of ARM v8(64/32 bit), Broadcom BCM 2837 System on chip used along with the CPU of $1.2 \mathrm{GHz} 64 / 32$ bit quad core ARM cortex A53. The RAM of Raspberry Pi $3 B$ is $1 G B$ and the storage is in the form of micro SD card. It has an additional feature of Wi-Fi and Bluetooth as compared to other versions of Raspberry $\mathrm{Pi}[4]$.

The features of RPi 3 includes 1.2GHz, 64-bit quad-core processor, 40 pin extended GPIO, 802.11bgn wireless LAN, WIFI, Bluetooth 4.1 connectivity, 1GB RAM, 4USB Ports, CSI camera port, Micro SD port, full size HDMI, and Micro USB Power source. In our project Keyboard, Mouse, Camera cables are connected to the 3 USB ports. HDMI cable is connected to the HDMI output, 1GB micro SD card with installed NOOBS[4].

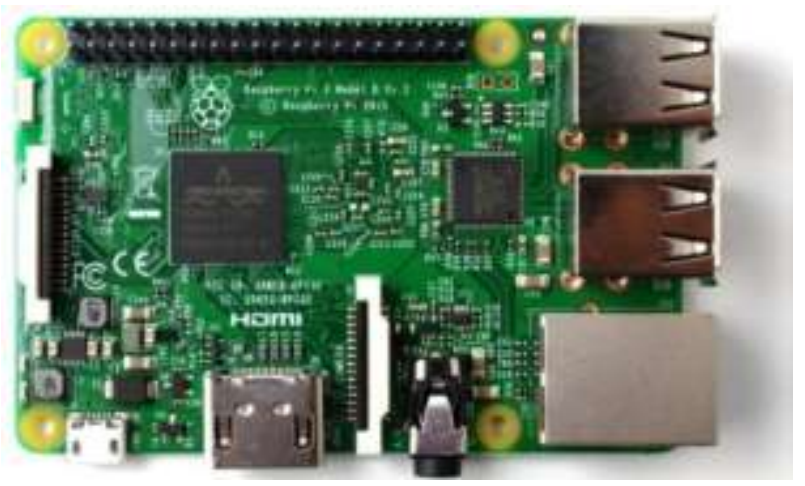

Fig 1: Raspberry Pi 3

\section{B. SD Card}

A SD card of 16/32 GB is used for the purpose of serving as memory for the Raspberry Pi, The OS is installed on this SD card.

\section{USB Camera}

A USB camera is used to capture the images. The USB camera is connected to the Raspberry Pi USB port.

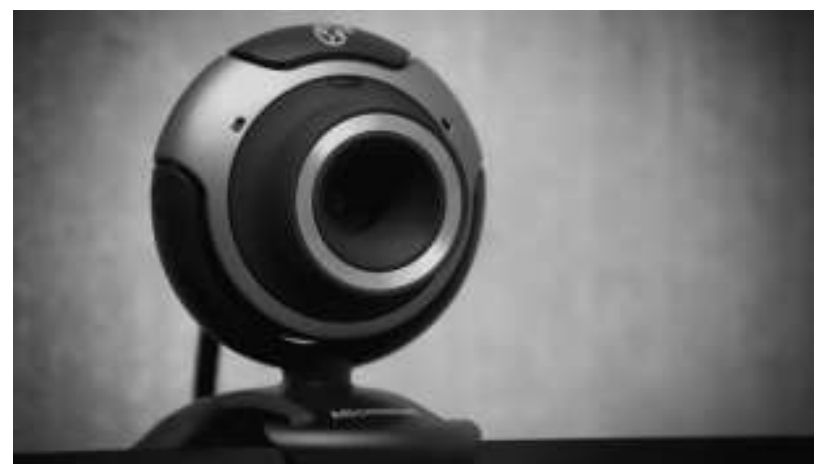

Fig 2: USB Camera

\section{Aux Speaker}

An aux speaker is used to inform the person trying to access the lab that whether he/she is allowed to enter the lab or not. 


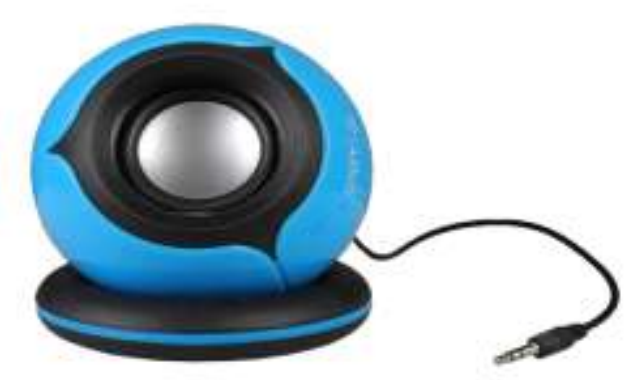

Fig 3: Aux Speaker

\section{E. Motor/Lock}

A motor/lock is used to open the door and allow the entry to the authorized person.

\section{SOFTWARE DESCRIPTION}

\section{A. Python IDLE}

IDLE is an integrated development environment for editing and running python2.x or python 3 programs. Where we can see or check the output.

\section{B. Raspbian OS}

Raspbian is a free operating system which is used run applications. To run our applications install the Raspbian OS. Raspbian OS is a good OS for RPi 3.

\section{NOOBS}

NOOBS -New Out Of Box Software is an installation manager for the Raspberry Pi. We install this manager in the SD card of Raspberry Pi.

\section{Python}

Python is a programming language. Which has easy syntaxes to read that allows fewer lines of code to the programmers. This language is also suitable for other customized applications.

\section{E. VNC Viewer}

VNC viewer is a software which allows us wireless access to our RPi. We can access and control the RPi through our laptop or mobile using the VNC Viewer.

\section{F. OpenCV}

OpenCV (Open Source Computer Vision Library) is a programming library consisting of functions mainly aimed towards real-time computer vision and is developed by Intel, it had been supported by Willow Garage then Itseez (later acquired by Intel). The library is cross-platform and free to be used under the open-source BSD license.

\section{G. RPi.GPIO}

RPi.GPIO is a Python library which allows the RPii to interact with circuits.

\section{H. pyttsx3}

pyttsx3 is a Python library for performing Text to Speech operations in Python.

\section{AWS Cloud}

There are two services of AWS which are used in our project, S3(Simple Storage Service) and Amazon Rekognition. S3 is used for the purpose of creating the database of images and Amazon Rekognition is required for the purpose of face recognition[7]-[8]. 


\section{WORKING OF THE PROPOSED SYSTEM}

In our proposed system the person trying to access the lab is authenticated by face recognition. Raspberry Pi is used for the purpose of automation. The camera is connected to Raspberry Pi USB port and it captures the image of the person trying to

gain access to the lab(camera captures the image only when the switch mounted near the gate is pressed). The image captured by the camera is then compared with the images in the database, if the person is authenticated then a node in the lab is assigned to the person. Authenticated person is informed about the assigned node through the speaker and the gate will be unlocked, also the appliances(fan and light) near the assigned node will be turned on automatically and also the assigned node will be provided the power. As soon as the user is logged out of the session node shuts down and the nearby appliances are also turned off automatically.

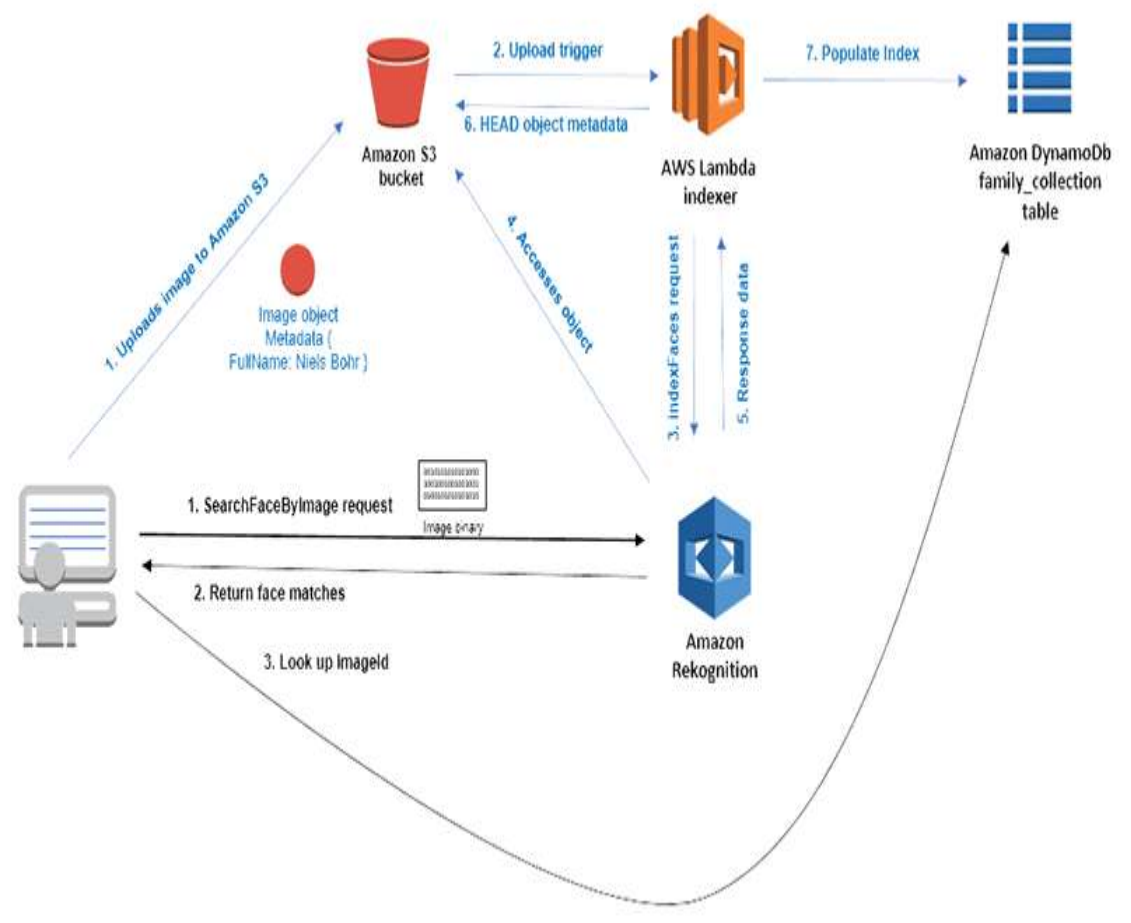

Fig 4: Working of Face Recognition System

The algorithm of the proposed system is as follows.

1. Write Raspbian OS in to the SD card and fix the card into the SD slot

2.Enable the VNC Viewer and Camera in the RPi configurations.

3. Install all the open CV libraries into the Raspberry Pi

4. Fix the entire hardware setup.

5. Create the image database of the authorised people with their names using the AWS S3(Simple Storage Service) and index the faces after the uploading of images is completed.

6. When a person tries to access the lab, check whether the lab is full or not, if the lab is not full then capture the image of that person otherwise tell that person to wait.

7.Compare the captured image with the image database using Amazon Rekognition..

8.If the person is authorised then move to step 8, otherwise tell the person that he is not allowed to access the lab.

9.Unlock the door and assign a node to the person[9].

10.Inform the person about the assigned node number. 
11. Unlock the door for that person.

12.Provide power to the node and nearby electrical

Appliances(fan and light) using Raspberry Pi.

13. When the person exits the lab, deassign the node assigned to that person.

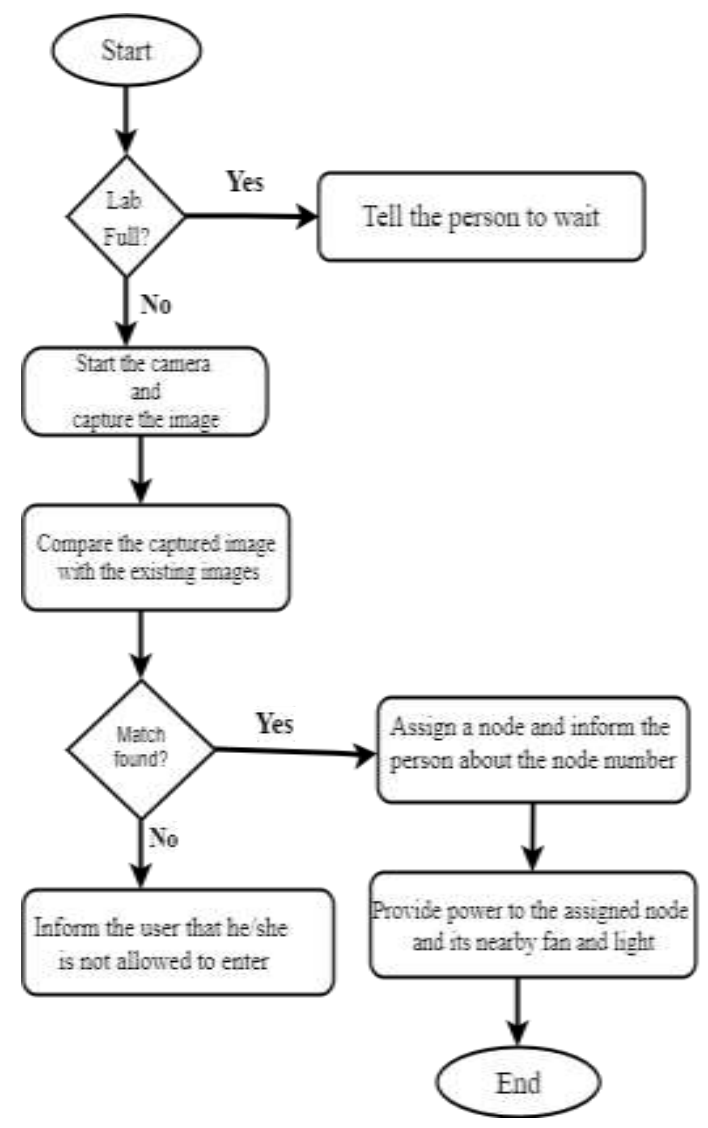

Fig 5: Flowchart of the proposed system

\section{RESULTS}

First the face database of a person is added onto the cloud using AWS S3, 


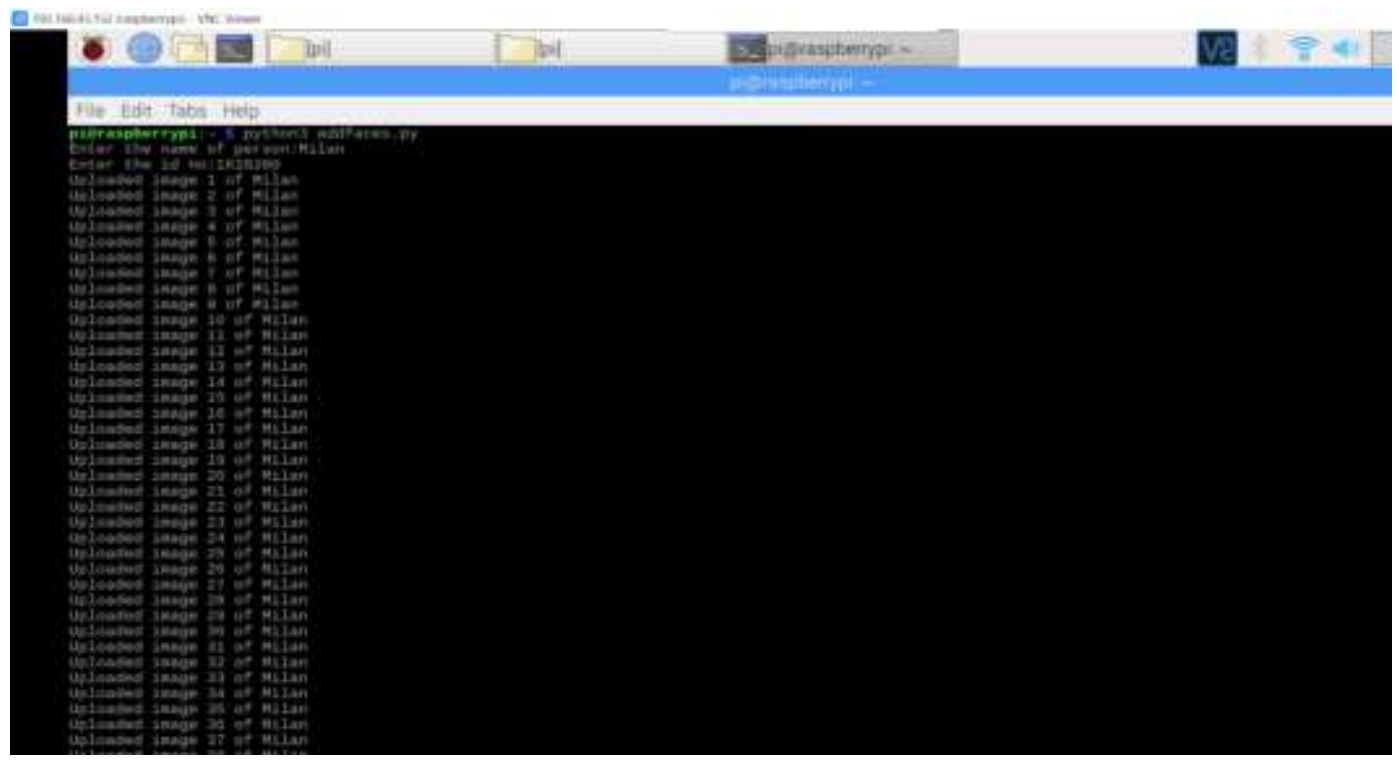

Fig 6: Adding a new face to database

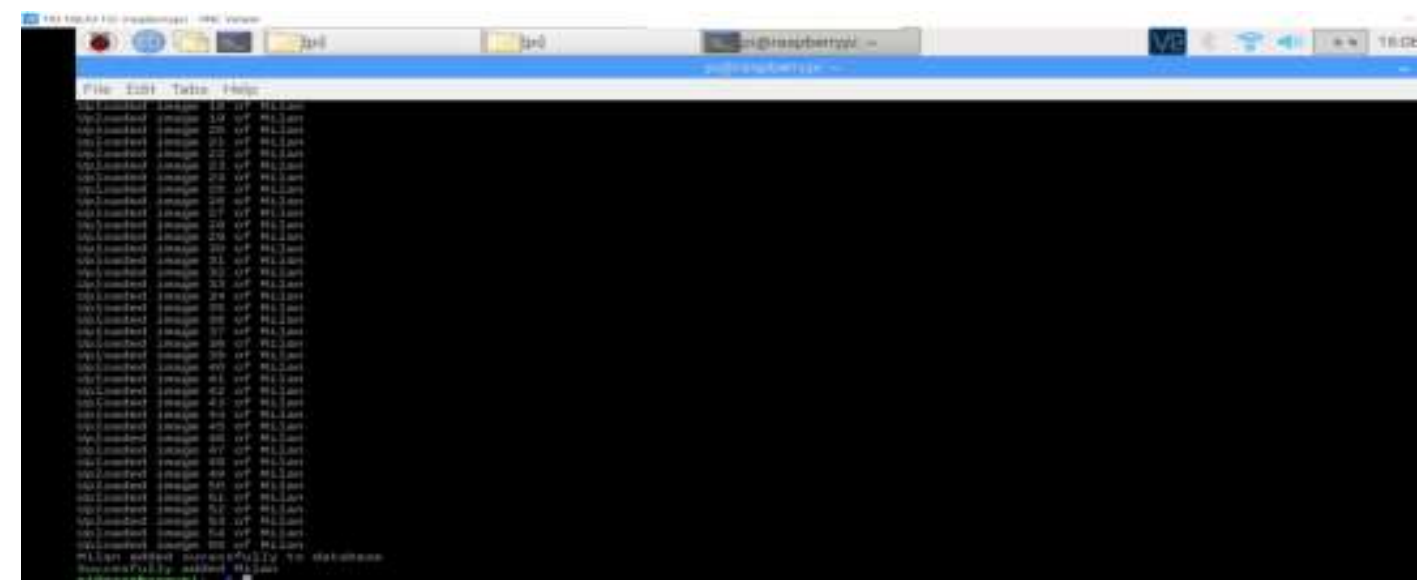

Fig 7: Images of a student added successfully to database

A folder with the name and ID number of the person is created in the database,

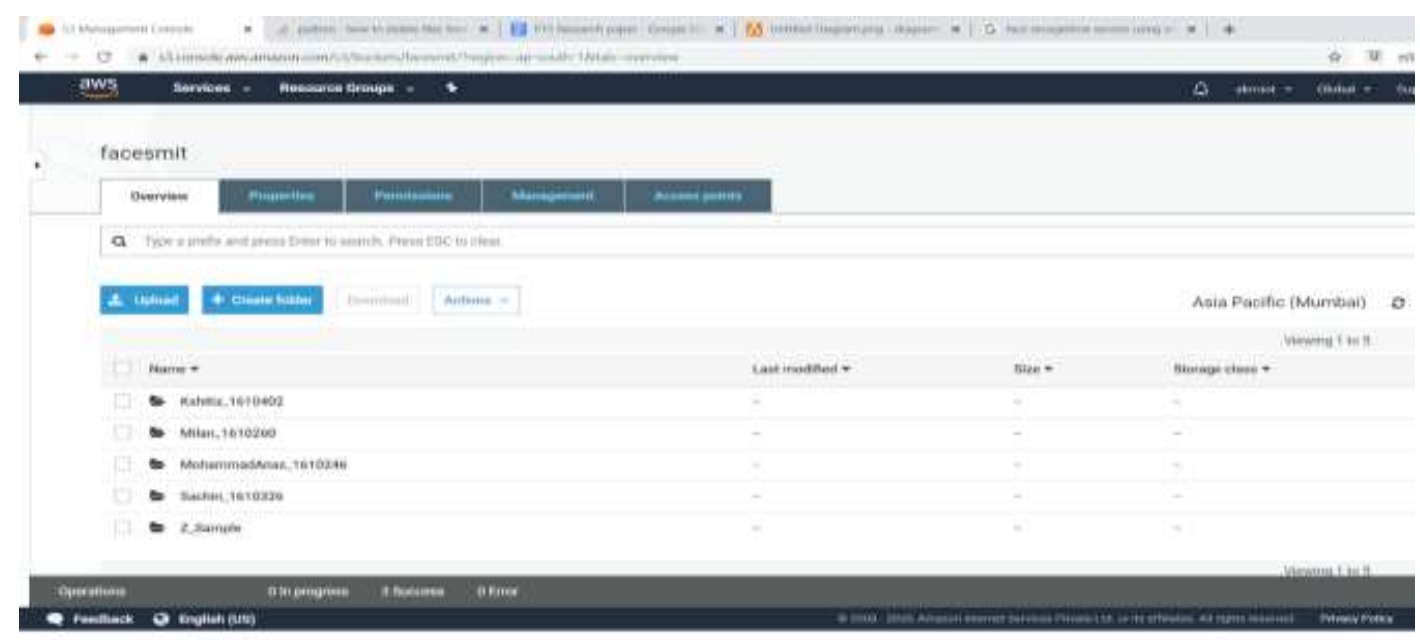

Fig 8: Image Database of each individual

After addition of a new person, we indexed the faces, 


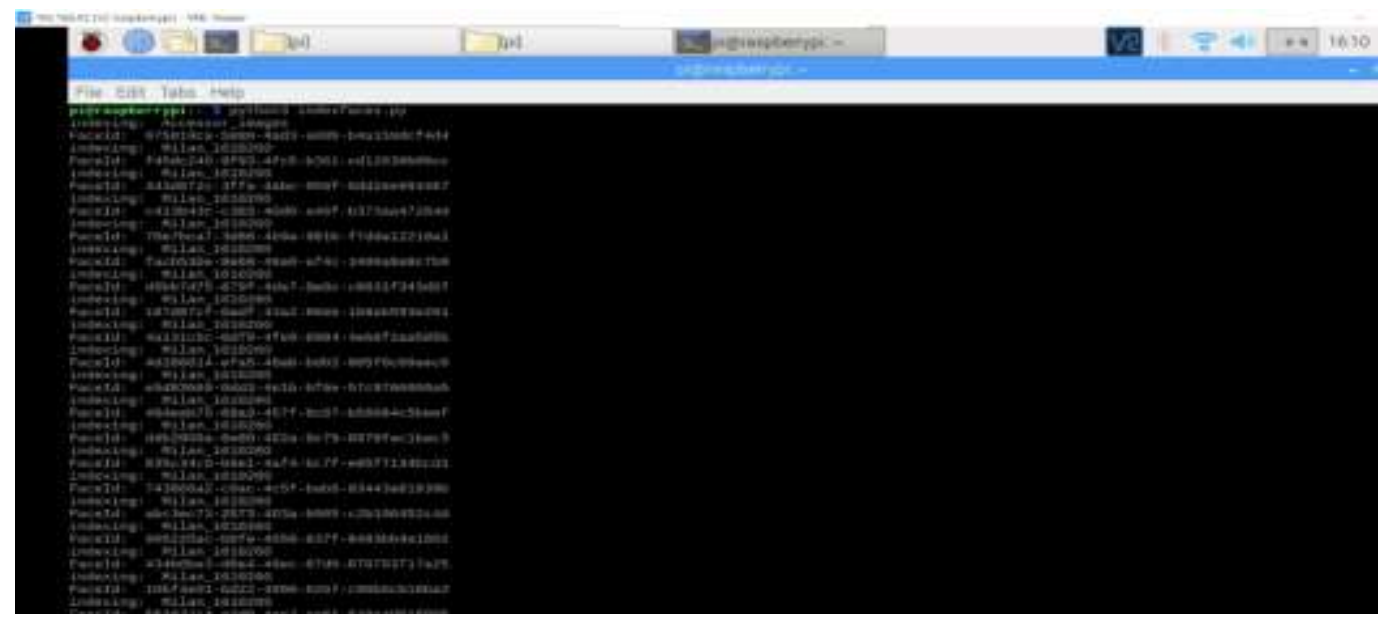

Fig 9: Indexing the faces

After indexing, the person is ready to be recognised and to be assigned a node. The name of the person is returned by the Amazon Rekognition and the person will be informed about the assigned node by the speaker.

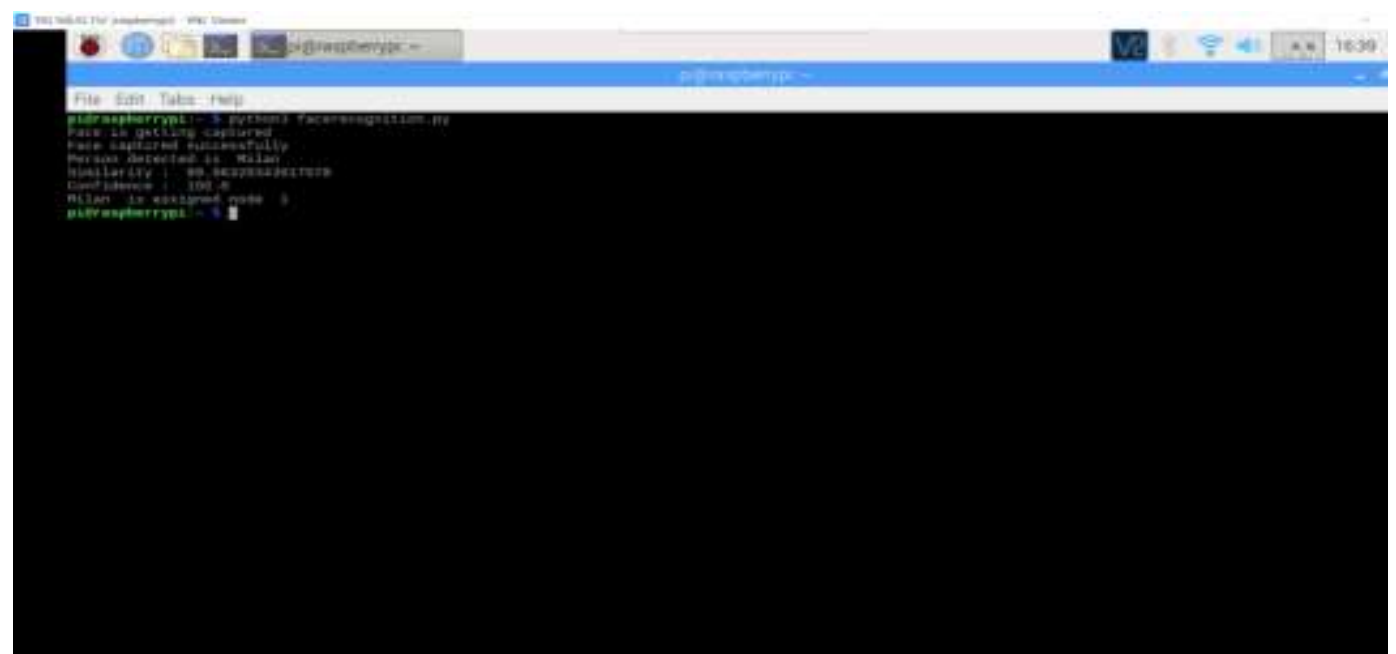

Fig 10: The person is recognised and informed about the assigned node(using speaker)

\section{CONCLUSION}

The usage of a facial recognition system with Raspberry Pi and AWS cloud can make the system littler, lighter and work successfully utilizing lower control use, so it is more convenient than the PC based face recognition system. It is based on open source software i.e Linux. Also, the announcement is done using the speaker so there is no need to design a UI for the user. The power backup for the smooth and continuous functioning of the system can be provided using power bank in case of power failure[10]. The power bank is used to power the Raspberry Pi so there is less chance to slow down the system.

This development scheme is cost effective, reliable and highly scalable and Raspberry pi takes less power and provides enough flexibility to suit the requirements.

The use of modern technologies such as cloud and IoT also makes this lab the lab of the future. In future we may expect similar things to become a part of our daily lives. As the technological advancements will take place and hardware systems will become better, we may also expect the processing to be faster.

\section{FUTURE WORK}

- If an unauthorised person tries to access the door, the system will send an Email to the admin regarding the same.

- A LCD display can be deployed to make the system more user -friendly. 
- Highly secure protocols can be deployed to ensure there is no security breach.

- Admin can add the holiday calendar to ensure that no person can access the lab on holidays.

- Two step verification process can also be added in which the person will be required to enter the OTP received on his mobile phone.

\section{REFERENCES}

[1] A. Kumar, P. S. Kumar and R. Agarwal, "A Face Recognition Method in the IoT for Security Appliances in Smart Homes, offices and Cities," 2019 3rd International Conference on Computing Methodologies and Communication (ICCMC), Erode, India, 2019, pp. 964-968, doi: 10.1109/ICCMC.2019.8819790.

[2] Anjali Patel, Ashok Verma, IOT based Facial Recognition Door Access Control Home Security System, International Journal of Computer Applications (0975 - 8887), Volume 172 - No.7, August 2017

[3] D. NarendarSingh,M. Kusuma Sri, K. Mounika, IOT Based Automated Attendance with Face Recognition System, International Journal of Innovative Technology and Exploring Engineering (IJITEE), ISSN: 2278-3075, Volume-8, Issue-6S4, April 2019

[4] Gubbi, Jayavardhana(2013) Internet of Things (IOT): A vision, architectural elements, and future directions. Future Generation Computer Systems 29.7: 1645-1660.

[5] Hassan,H., Bakar,R.A., Mokhtar,A.T.F, "Face recognition based on auto-switching magnetic door lock system using microcontroller",IEEE-International Conference on System Engineering and Technology (ICSET), 2012

[6] I. Gupta, V. Patil, C. Kadam and S. Dumbre, "Face detection and recognition using Raspberry Pi," 2016 IEEE International WIE Conference on Electrical and Computer Engineering (WIECON-ECE), Pune, 2016, pp. 83-86, doi: 10.1109/WIECONECE.2016.8009092.

[7] Glenn J. Pulvera, Assessment of Cloud Computing Capabilities of State Universities and Colleges (SUC), International Journal of Science and Management Studies (IJSMS), E-ISSN: 2581-5946, Volume: 02 Issue: 02, March to April 2019

[8] M. Sahani, C. Nanda, A. K. Sahu and B. Pattnaik, "Web-based online embedded door access control and home security system based on face recognition," 2015 International Conference on Circuits, Power and Computing Technologies [ICCPCT-2015], Nagercoil, 2015, pp. 1-6, doi: 10.1109/ICCPCT.2015.7159473.

[9] Md. Naseem Uz Zaman Chowdhury, Md. ShibleeNooman ,SrijonSarker,Access Control of Door and Home Security by Raspberry Pi Through Internet,International Journal of Scientific \& Engineering Research, Volume 4, Issue November-2013 ISSN $2229-5518$

[10] T. Soyata, R. Muraleedharan, C. Funai, M. Kwon and W. Heinzelman, "Cloud-Vision: Real-time face recognition using a mobilecloudlet-cloud acceleration architecture," 2012 IEEE Symposium on Computers and Communications (ISCC), Cappadocia, 2012, pp. 000059-000066, doi: 10.1109/ISCC.2012.6249269. 